\title{
High-Thick Thermal Reflow Process Using Vacuum-assisted Micromolding for Submillimeter Microvalves
}

\author{
Takaaki Abe, ${ }^{1}$ Shunya Okamoto, ${ }^{1}$ and Yoshiaki Ukita ${ }^{2 *}$ \\ ${ }^{1}$ Department of Engineering, Integrated Graduate School of Medicine, Engineering, and Agricultural Sciences, \\ University of Yamanashi, 4-3-11 Takeda, Kofu, Yamanashi 400-8510, Japan \\ ${ }^{2}$ Graduate Faculty of Interdisciplinary Research, University of Yamanashi, \\ 4-3-11 Takeda, Kofu, Yamanashi 400-8510, Japan
}

(Received April 5, 2021; accepted July 29, 2021)

Keywords: microfluidics, nano/microengineering, reflow process, microvalves

We report a reflow process for forming rounded channels of submillimeter height. In this method, a hot-melt glue comprising primarily of an ethylene-vinyl acetate (EVA) copolymer is patterned onto a substrate via vacuum-assisted micromolding (VAM) in capillaries using polydimethylsiloxane (PDMS) molds. This method enables a submillimeter channel structure to be fabricated using a thermoplastic resin. Subsequently, by heating the mold, rounded crosssectional channels with a height of $100 \mu \mathrm{m}$ order can be fabricated, which is difficult to achieve in the conventional reflow process using positive resists. By observing the cross-sectional shape of the reflowed mold, it is confirmed that the cross-sectional shape of the channels can be controlled by changing the heating time. In addition, the method offers flexibility in terms of the channel layout. As an application to microfluidic devices, we fabricate and evaluate a microperistaltic pump comprising three microvalves using a typical hot plate and a small vacuum pump. In addition, the PDMS mold and fabricated glue mold can be used repeatedly. In general, the proposed process is a simple and inexpensive means of fabricating micropumps and valves that can handle large objects such as cell tissues.

\section{Introduction}

Soft lithography for the fabrication of polydimethylsiloxane (PDMS) microfluidic devices has contributed significantly to the development of microfluidics. ${ }^{(1,2)}$ Microvalves fabricated using this technique are essential components for fluid control in microfluidic systems. ${ }^{(3,4)}$ In particular, normally open valves driven by pneumatic pressure are used extensively in microfluidic systems because of their simple structure and easy implementation in devices. The flow channel is closed by pneumatic pressure owing to the deformation of the valve's elastomeric diaphragm. This principle has been applied to sorters, ${ }^{(5)}$ mixers, ${ }^{(6)}$ and pumps ${ }^{(7)}$ by combining several valves. When implementing these valves, the cross section of the channel must be round to completely close and seal the channel. ${ }^{(3,8)}$ Since the size of objects manipulated by microfluidic valves can be as large as several hundred micrometers, it is necessary to open and close a flow

*Corresponding author: e-mail: yukita@yamanashi.ac.jp

https://doi.org/10.18494/SAM.2021.3379 
channel large enough to transport them. In addition, complex and expensive device fabrication methods offset the advantages of soft lithography on a rapid prototyping, so simple and low-cost methods are required.

Thermal reflow is a technique used to fabricate micrometer-scale curved surface structures. ${ }^{(9-11)}$ In particular, the reflow process using a positive photoresist is often used to fabricate rounded cross-sectional channel structures. ${ }^{(6,8,12-14)}$ In this method, a positive photoresist pattern on a substrate is heated to create a rounded cross section by a shape change that causes the resist to melt. However, when using a positive photoresist, the channel height achieved by spin-coating is limited to several tens of micrometers owing to the limitation of the resist thickness. ${ }^{(8)}$ Meanwhile, when managing relatively large objects such as cultured cell tissues, channel heights of $100 \mu \mathrm{m}$ or more are required. ${ }^{(15,16)}$ A different method must be adopted to fabricate larger channels for these objects. Examples of methods that can be used to fabricate rounded structures on the scale of several hundred micrometers include grayscale lithography ${ }^{(17)}$ and micromilling. ${ }^{(15,18)}$ Grayscale lithography can be used to fabricate 3D structures but requires expensive optical equipment. In addition, micromilling requires highprecision milling machines, and cutting marks on the channel surface and edge burrs are difficult to eliminate. Other methods present challenges such as height constraints $(<150 \mu \mathrm{m})$, the necessity for surface treatment on the mold for the double casting of PDMS, and complex valve structures. ${ }^{(19,20)}$ Therefore, there is no simple and low-cost method for fabricating valves with a channel height of several hundred micrometers.

Herein, we propose a simple and low-cost method for fabricating molds that can be reflowed using vacuum-assisted micromolding (VAM), ${ }^{(21)}$ which originates from micromolding in capillaries, ${ }^{(22,23)}$ where a vacuum force is used instead of the capillary force. This method has been used to pattern microstructures by filling microchannels in PDMS chips with a material such as a polymer precursor using a vacuum. To date, the patterning of ceramics ${ }^{(24,25)}$ and ultraviolet (UV) curing resin ${ }^{(26,27)}$ on substrates has been reported. We patterned an ethylene-vinyl acetate (EVA)-based hot-melt glue on a silicon wafer via VAM. This allowed us to replicate a relatively large channel structure (up to hundreds of micrometers in height) made of SU-8 negative photoresist using a thermoplastic resin. Although SU-8 can be used to fabricate thick structures, it cannot be reflowed directly because it is a thermosetting resin. However, by our method, thick molds that can be thermally reflowed similarly to a positive photoresist can be fabricated. To demonstrate this method, we fabricated a straight channel with a rounded cross section and evaluated the reproducibility of the cross-sectional shape by reflowing the mold at different heating times. Subsequently, to demonstrate the patterning ability of complex curved channels required in microfluidic systems, we evaluated the shape change of the cross section of the straight or curved section. Moreover, as an application of this method, microvalves and microperistaltic pumps were fabricated, the closing pressure of the valve was evaluated, and high-flow-rate micropumping was demonstrated. 


\section{Materials and Methods}

\subsection{Patterning and thermal reflow process of EVA hot-melt glue}

Figure 1 shows an overview of VAM. A PDMS mold, which was cast using an SU-8 master mold, was attached to a silicon wafer on a hot plate, which was set to $200{ }^{\circ} \mathrm{C}$. A solid EVA glue (K-13P-12, T-098, Daiso Industries Co., Ltd., Japan) was placed in one of the reservoirs and allowed to stand for about $1 \mathrm{~min}$ to melt. No spontaneous flow into the channel by capillary force occurred, and the glue was injected into the channel of the PDMS mold by depressurizing the opposite port using a diaphragm pump (DAP-6D, ULVAC KIKO Inc., Japan), and the minimum pressure at depressurization was $-94 \mathrm{kPa}$. The diaphragm pump was stopped when the glue injection was completed, and the wafer and PDMS mold were cooled. Because the PDMS mold was attached to the wafer in an expanded state owing to the heating by the hot plate, it might have spontaneously peeled off from the wafer because of shrinkage during cooling and hence damage the glue before solidification. Therefore, a polyethylene terephthalate sheet (VF-1410N, KOKUYO Co., Ltd., Japan) was attached to the top surface of the PDMS mold immediately before cooling to prevent peeling due to shrinkage. After cooling the wafer and PDMS mold to room temperature, the PDMS mold was peeled off. EVA glue shows strong adhesion to the surface of silicon wafers but little adhesion to PDMS. Owing to this property, the molded glue can be easily released from the PDMS mold. The EVA glue mold fabricated via VAM exhibited a rectangular cross section owing to the transfer of the structure of the SU-8 flow channel. By reflowing this glue mold on a hot plate, channels with a rounded cross section were fabricated.

\subsection{Mold and device fabrication}

Figure 2 illustrates the process involved in the proposed method. The PDMS mold was fabricated from the SU-8 master mold [Figs. 2(a)-2(c)]. The SU-8 master mold was fabricated by
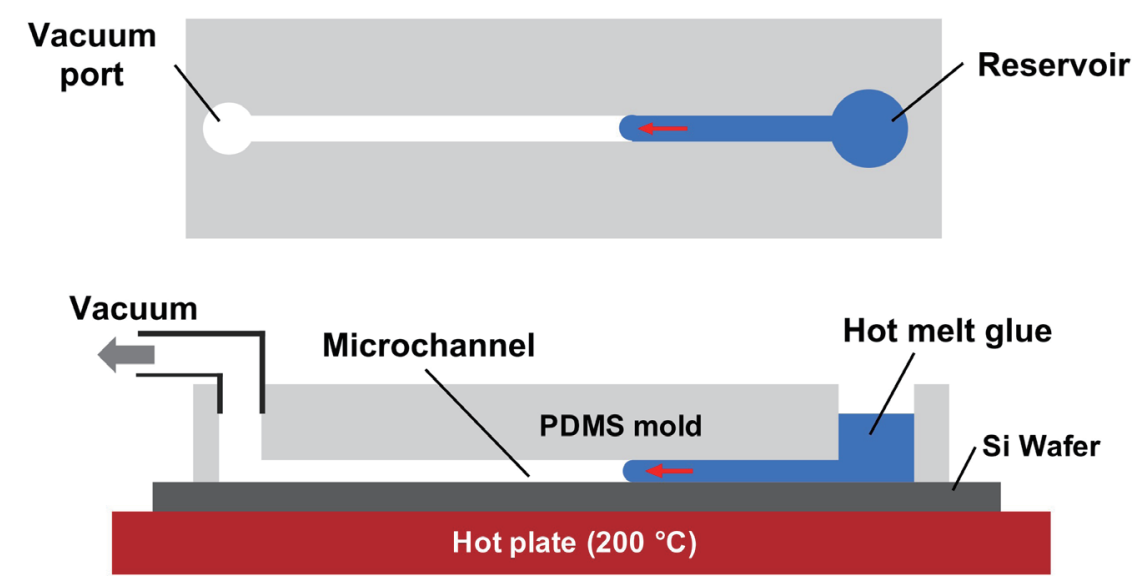

Fig. 1. (Color online) Overview of VAM in capillaries 


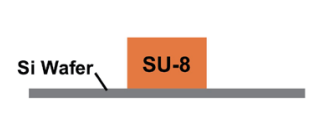

(a)

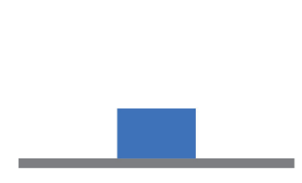

(e)

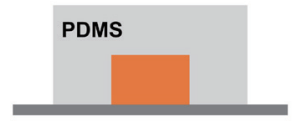

(b)

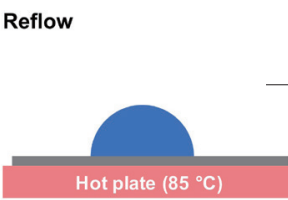

(f)

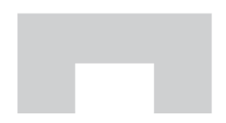

(c)

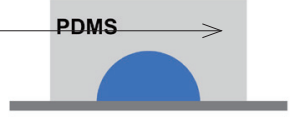

$(\mathrm{g})$

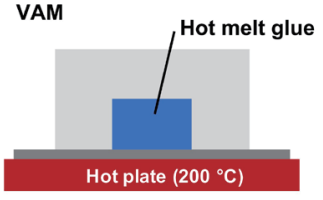

(d)

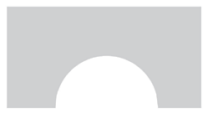

(h)

Fig. 2. (Color online) Procedure of mold fabrication and reflow process using VAM. (a) Fabrication of master mold by photolithography. (b, c) Fabrication of PDMS mold for VAM from master mold. (d, e) Patterning of EVA hot-melt glue via VAM. (f) Heating of glue mold using hot plate. (g, h) Casting PDMS with glue mold after reflow.

patterning a negative photoresist (SU-8 2100, Nippon Kayaku Co., Ltd., Japan) on a silicon wafer via photolithography. SU-8 was spin-coated on a silicon wafer and soft-baked at $95{ }^{\circ} \mathrm{C}$ for $60 \mathrm{~min}$. The wafer was exposed to $250-500 \mathrm{~mJ} / \mathrm{cm}^{2}$ of UV light through a photomask with a printed channel pattern. Subsequently, it was developed using the SU-8 developer (Nippon Kayaku Co., Ltd., Japan) after a post-bake for $5 \mathrm{~min}$ at $95{ }^{\circ} \mathrm{C}$. The PDMS molds and devices were fabricated using soft lithography. ${ }^{(28)}$ A silicon rubber sheet (6-612-04, AS ONE Corporation, Japan) of $2 \mathrm{~mm}$ thickness was attached to the SU-8 mold as a spacer to adjust the device thickness. PDMS (Sylgard184, The Dow Corning, USA) was mixed with a curing agent at a ratio of 10:1 and applied onto the mold. After defoaming it in a vacuum chamber for $30 \mathrm{~min}$, it was cured by heating in an oven at $75{ }^{\circ} \mathrm{C}$ for 90 min. After curing, the PDMS was peeled and cut, and a reservoir hole for the glue was punched out using a 4-mm-diameter punch. The hard baking of the PDMS mold used in this study was omitted. This is because if the adhesion between the wafer and the edge of the channel is reduced by deformation due to heating around the channel, the melted glue may leak from the channel of the PDMS mold. The glue mold was fabricated from the PDMS mold using VAM [Fig. 2(d)]. A rounded cross-sectional channel was fabricated by the reflow of the mold on a hot plate heated to $85^{\circ} \mathrm{C}$ [Figs. 2(e) and 2(f)]. Since the rate of the shape change of the cross section increases in proportion to the reflow temperature, the effect of the error in the heating time for shape control increases with the temperature. Therefore, to ensure the reproducibility of the cross-sectional shape, the reflow temperature was set near the melting temperature of the glue. Subsequently, PDMS was cast from the glue mold for device fabrication and evaluation [Figs. 2(g) and 2(h)].

\subsection{Evaluation of cross section of channels and patterning ability}

To evaluate the cross-sectional shape of the channels, straight channels with widths of 100 , 300 , and $800 \mu \mathrm{m}$ and a length of $40 \mathrm{~mm}$ were used. SU- 8 master molds were fabricated using the 
procedure described in Sect. 2.2. The resist thicknesses of the three molds corresponding to the abovementioned channels were 48.8, 155.1, and $494.1 \mu \mathrm{m}$, respectively. PDMS molds were cast from SU-8 master molds, and the EVA glue was patterned on the silicon wafers by VAM using PDMS molds. Subsequently, the glue molds were reflowed on a hot plate, and a replica was fabricated using PDMS soft lithography. The cross section of the replica was observed under a microscope. The contact angle of the channel was calculated from the width $w$ and height $h$ of the cross-sectional image of the PDMS replica. The contact angle $\theta$ was calculated using the following equation.

$$
\theta=2 \tan ^{-1}\left(\frac{h}{w / 2}\right)
$$

Figure 3(a) shows the channel structure used to demonstrate the patterning ability. Channel patterns with different radii of curvature were fabricated on the same substrate and reflowed. The radius of curvature was set to $r_{n}=n w$, which is a constant multiple of the design value of the channel width $w$. The resist thicknesses of the SU-8 master molds were 49.6 and $421.4 \mu \mathrm{m}$. The curve pattern was evaluated by measuring the channel widths of the PDMS replicas fabricated using reflowed glue molds. During the reflow of the glue molds, the edge inside the curved part advanced toward the inside of the curve owing to the melting and deformation of the glue. Assuming that the cross-sectional area of the channel was preserved, the height of the channel decreased [Fig. 3(b)]. The shape change in the curved channel was evaluated by measuring the difference between the widths of the straight and curved channels.

\subsection{Measuring valve opening and flow rate}

Figure 4 shows the structure of the microperistaltic pump. This pump comprised three layers: the channel layer, diaphragm, and pneumatic layer. The design widths of the microchannels were 100,300 , and $800 \mu \mathrm{m}$, and the widths $w_{v}$ of the pneumatic chambers were 250,800 , and

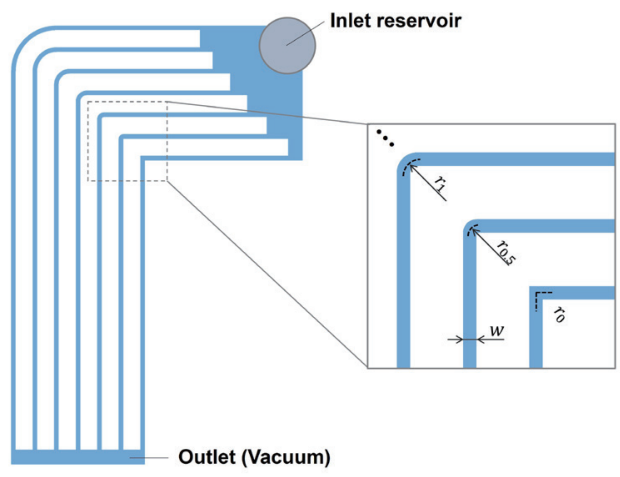

(a)
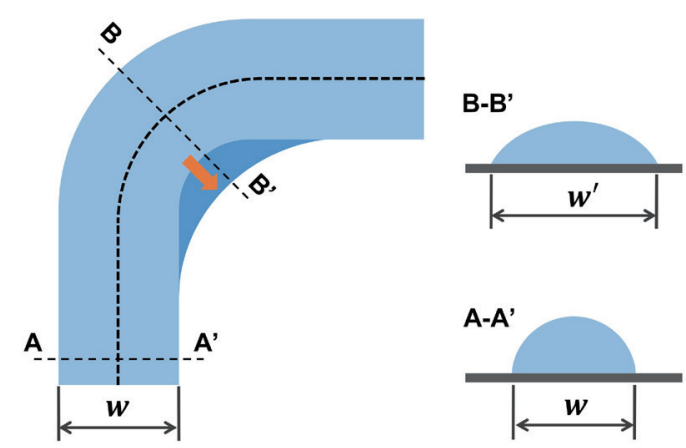

(b)

Fig. 3. (Color online) (a) Channel structure of mold. (b) Method for evaluating curve. 


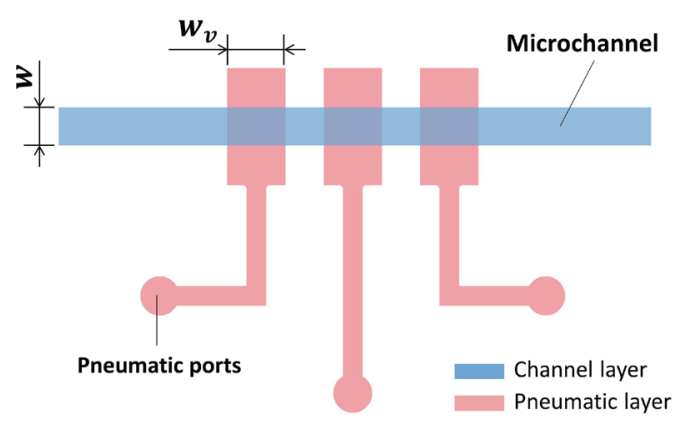

(a)
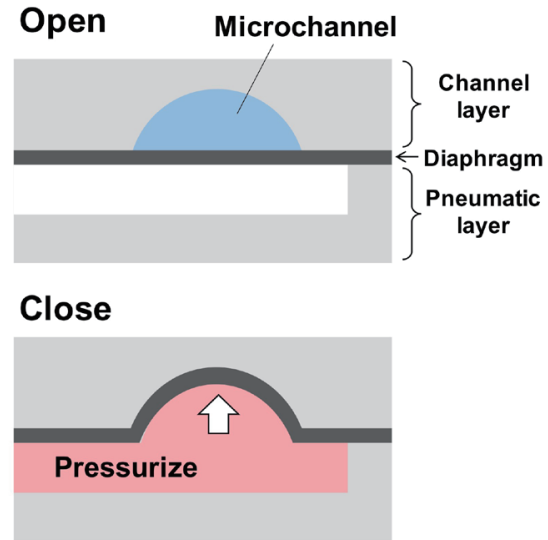

(b)

Fig. 4. (Color online) (a) Structure of microperistaltic pump. (b) Cross-sectional view of valve.

$1500 \mu \mathrm{m}$, respectively. These straight channels were patterned on silicon wafers via VAM and reflowed on a hot plate at $85^{\circ} \mathrm{C}$ for 30,60 , and $300 \mathrm{~s}$ for the widths of 100,300 , and $800 \mu \mathrm{m}$, respectively. After the mold was fabricated, a silicon rubber sheet of $3 \mathrm{~mm}$ thickness was attached to the channel layer molds, whereas one with a thickness of $2 \mathrm{~mm}$ was attached to the pneumatic layer molds as a spacer to adjust the device thickness. Subsequently, PDMS was cast using the same process as that used to fabricate the PDMS molds. The outer shape of the PDMS peeled from the mold was cut, and its chambers were punched out using a punch with a diameter of $8 \mathrm{~mm}$. The diaphragm was formed by spin-coating PDMS mixed at a ratio of 10:0.7 on the silicon wafer. It was cured by heating at $75^{\circ} \mathrm{C}$ for $90 \mathrm{~min}$. The thickness of the diaphragm was 26.2-28.5 $\mu \mathrm{m}$. The diaphragm and the pneumatic layer were irradiated with air plasma and bonded by heating on a hot plate at $75{ }^{\circ} \mathrm{C}$ for $15 \mathrm{~min}$. This layer and the channel layer were bonded by the same method. The pressurization system of the micropump comprised a compressor, a solenoid valve (M3QB110-M5-C2H-8-4, CKD Corporation, Japan) to drive the diaphragm, and an Arduino microcontroller (Arduino Uno, Arduino SRL, Italy) to operate the valve. The pneumatic chamber was filled with Milli-Q water to prevent the diaphragm from deflecting. For the fluorescence observation of the valve, the microchannel was captured using an sCMOS camera (ORCA-flash4.0, C11440-22CU, Hamamatsu Photonics K.K.) attached to a microscope (IX71, Olympus Corporation, Japan). The microfluidic channels were filled with fluorescein (F6377-100G, Sigma-Aldrich Co., USA) diluted to $1 \mathrm{mM}$ in Milli-Q water. To observe the channel, which had a width of $100 \mu \mathrm{m}$, a 10× objective lens (UPLANFLN10x, Olympus Corporation, Japan), a mirror unit (U-MNIBA2, Olympus Corporation, Japan), and a filter (25ND25, Olympus) were used. For the other channels, a $4 \times$ objective lens (UPLANFL4x, Olympus) and a mirror unit (U-MWIB2, Olympus) were used. When measuring the flow rate, the channel was filled with Tween 20 (170-6531, Bio-Laboratories, Inc., USA) and Victoria Blue B (228-00222, Wako Pure Chemical Industries Ltd., Japan) diluted in Milli-Q water to $0.05 \%$ $(\mathrm{v} / \mathrm{v})$ and $0.1 \mathrm{mM}$, respectively. Polyethylene beads (REDPMS-1.085 27-32 $\mu \mathrm{m}$, Cospheric Microspheres, USA) were used as a fluid tracer in the devices with $w=100$ and $300 \mu \mathrm{m}$. Beads 
measuring 90-106 $\mu \mathrm{m}$ (REDPMS-1.065 90-106 $\mu \mathrm{m}$, Cospheric Microspheres) were placed in the device with $w=800 \mu \mathrm{m}$. The beads were photographed during the pump operation, and the flow rate was calculated from the cross-sectional area of the channel and the number of beads that shifted. For the open and closed states of the valve, represented as open(0) and closed(1), respectively, the operating sequence was defined as (001)(100)(111). The operating frequency indicates the one-state transition period of the valve.

\section{Results and Discussion}

\subsection{Observation of channel cross section}

The contact angle between the channel and the substrate was measured to characterize the channel shape. For convenience of discussion, we refer to the channels as Types A, B, and C in order from the smallest to the largest cross-sectional area. The time from the start of depressurization until filling with glue to a certain distance from the channel is determined by the cross-sectional shape, glue viscosity, and depressurization pressure. ${ }^{(21)}$ The largest channel (Type C) required only a few seconds to fill the channel to a distance of $40 \mathrm{~mm}$, whereas the smallest channel (Type A) required approximately $10 \mathrm{~min}$ to fill the channel to a distance of 30 $\mathrm{mm}$. The measurement position of the channel cross section was taken near the center of the molded channel, i.e., $20 \mathrm{~mm}$ from the inlet for the 300 and $800 \mu \mathrm{m}$ channels and $15 \mathrm{~mm}$ from the inlet for the $100 \mu \mathrm{m}$ channel. Also, the longer and thinner the channel that is filled with the glue, the more the flow resistance increases, and thus the velocity of the glue decreases. In our experience, a channel with an equivalent diameter of about $30 \mu \mathrm{m}$ requires $2 \mathrm{~h}$ to fill to a distance of $30 \mathrm{~mm}$. In some cases, when the channel width was less than $30 \mu \mathrm{m}$, there were problems with the stability of the wetting behavior during reflow. After patterning, the glue molds were heated on a hot plate at $85{ }^{\circ} \mathrm{C}$. Figure 5 shows the Type $\mathrm{C}$ mold before and after

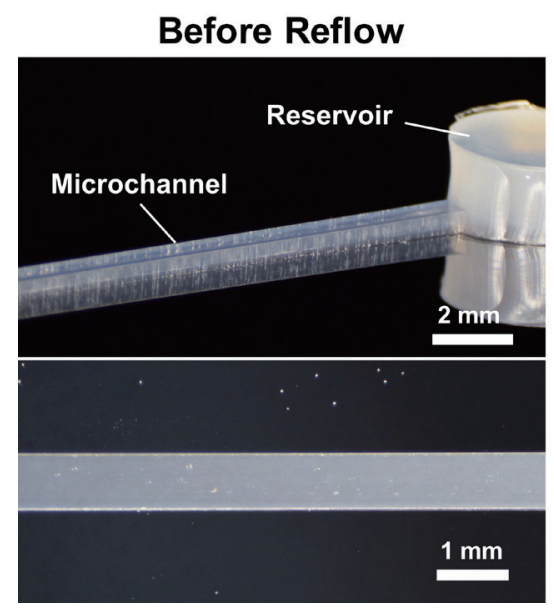

(a)

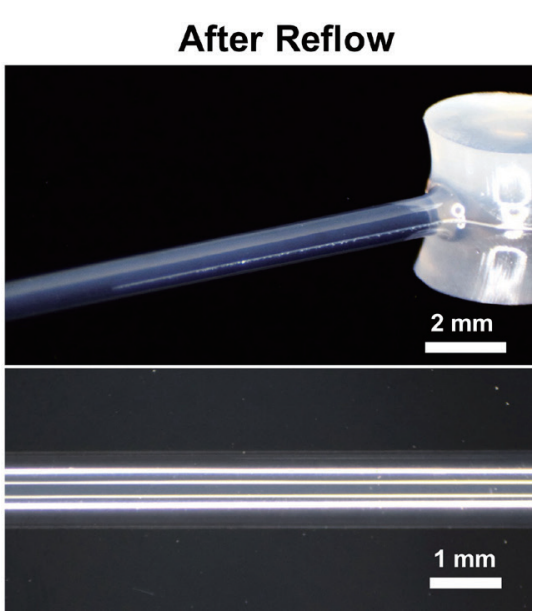

(b)

Fig. 5. (Color online) Glue mold before and after reflow. 
heating. As shown, the reflow process yielded a rounded cross section and a smooth surface. Figures 6(a) and 6(c)-6(h) show cross-sectional images of the PDMS replica fabricated using the glue molds. In this experiment, the curing temperature of the PDMS was set near the melting point of the glue, but the shape of the channel did not change upon heating at $75{ }^{\circ} \mathrm{C}$. This was

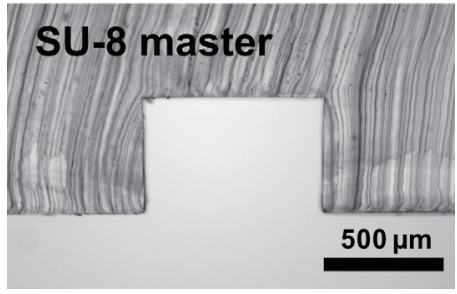

(a)

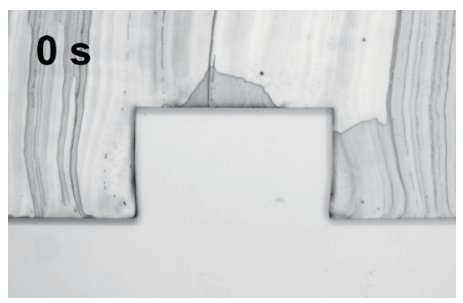

(c)

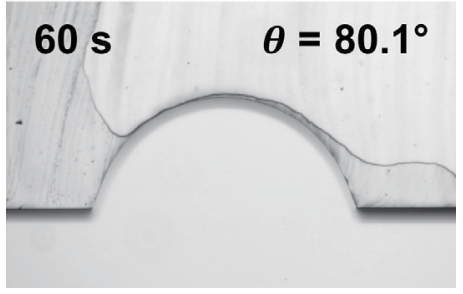

(f)

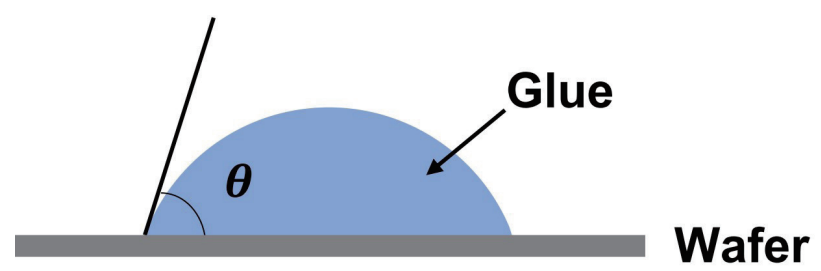

(b)

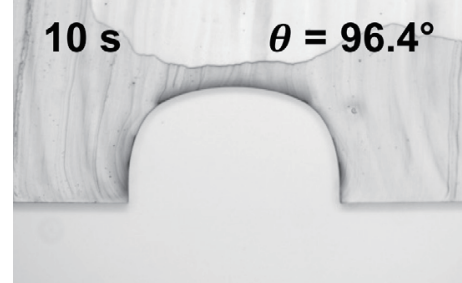

(d)

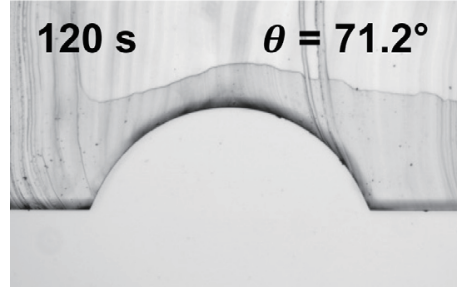

$(\mathrm{g})$

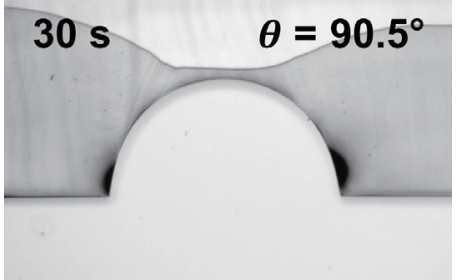

(e)

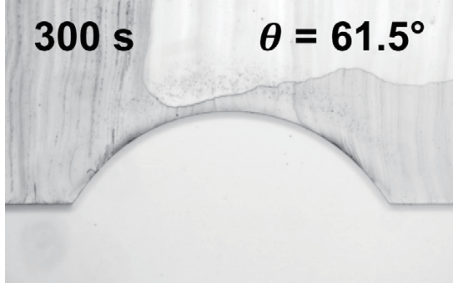

(h)

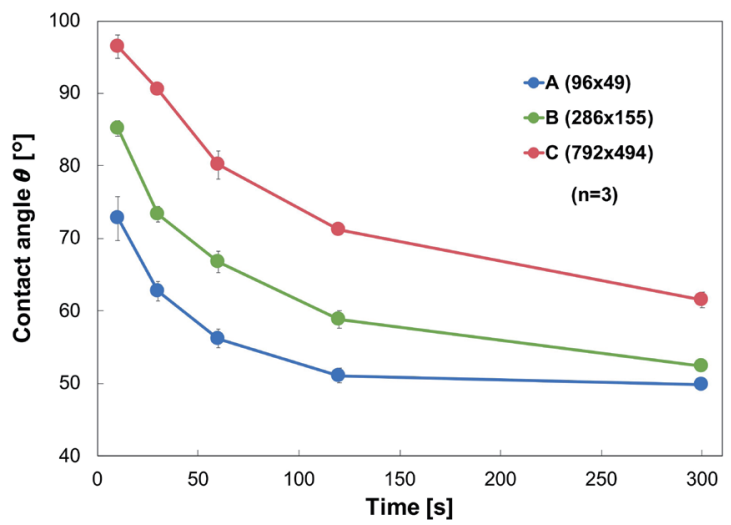

(i)

Fig. 6. (Color online) Cross-sectional shape of mold: (a) SU-8 master mold. (b) Definition of channel contact angle. (c) Glue mold before reflow. (d-h) Molds fabricated by reflow for 10-300 s. Contact angles shown in the upper right corner are average values of three experiments. (i) Channel contact angle vs reflow time. Numbers in the legend represent the dimensions (width $\times$ height) $[\mu \mathrm{m}]$ of the rectangular channel in the glue mold before reflow. Error bars show the standard deviation. 
confirmed by the fact that the channel edges and the roughness of the walls were maintained even after multiple casting of PDMS using a rectangular glue mold. However, the shape might change, depending on the environment in which heating is performed. In such a case, it may be possible to take countermeasures such as heating at a lower temperature by $5-10{ }^{\circ} \mathrm{C}$ and extending the curing time of PDMS. Figures 6(a) and 6(c) show the cross sections of the SU-8 mold and glue mold before reflow, respectively. The contact angle of the channel with the wafer was used to quantitatively evaluate the cross-sectional shape [Fig. 6(b)]. Figure 6(i) shows the transition of the contact angle with the heating time for the three types of mold. In addition, Table 1 shows the channel dimensions and the contact angles at each heating time, where the values for $0 \mathrm{~s}$ are the dimensions of the rectangular glue mold. It was observed that the contact angle decreased with increasing reflow time from the initial rectangular cross section and finally approached approximately $50^{\circ}$. With increasing size of the cross-sectional channel, the thermal energy required for the phase transition increased, and the top surface of the channel structure became farther from the heating surface of the hot plate. Therefore, the shape change of the channel proceeded slowly. The maximum channel height achieved in this experiment was $503 \mu \mathrm{m}$, which was for Type $\mathrm{C}$ with a heating time of $30 \mathrm{~s}$, and the width of this channel was 996 $\mu \mathrm{m}$. Among the three different types of channel, the maximum coefficient of variation (CV), indicating the variation in the contact angle, was approximately $4.2 \%$, confirming the high reproducibility of this process. This indicates that the cross-sectional shape of the glue mold can be controlled by varying the heating time. No shape changes or glue mold breakage was observed, even after more than five PDMS castings. In addition, no PDMS mold breakage was observed even after VAM was performed more than five times.

\subsection{Patterning of complex structures}

To demonstrate the ability of our proposed process to perform complex patterning, a channel structure that bends at right angles was fabricated. By measuring the difference in the widths of the curved and straight portions after reflow, it was determined whether the cross-sectional shape in the curved portion was retained. Figures 7(a) and 7(b) show a mold with a width of 800 $\mu \mathrm{m}$ before and after reflow, respectively. No leakage of glue into neighboring channels was observed. Figure 7(c) shows the error of the channel widths in the curved and straight portions with respect to the radius of curvature. When the radius of curvature was $w / 2$, the width of the curved portion increased by $25-30 \%$ compared with that of the straight portion. This indicates

Table 1

Channel dimensions and contact angles at each heating time. Error ranges show the standard deviation.

\begin{tabular}{|c|c|c|c|c|c|c|c|c|c|}
\hline \multirow{2}{*}{$\begin{array}{l}\text { Time } \\
\text { (s) }\end{array}$} & \multicolumn{3}{|c|}{ A $(96 \times 49)$} & \multicolumn{3}{|c|}{ B $(288 \times 155)$} & \multicolumn{3}{|c|}{$\mathrm{C}(792 \times 494)$} \\
\hline & Width $(\mu \mathrm{m})$ & Height $(\mu \mathrm{m})$ & $\theta\left(^{\circ}\right)$ & Width $(\mu \mathrm{m})$ & Height $(\mu \mathrm{m})$ & $\theta\left({ }^{\circ}\right)$ & Width $(\mu \mathrm{m})$ & Height $(\mu \mathrm{m})$ & $\theta\left(^{\circ}\right)$ \\
\hline 0 & $97.8 \pm 1.0$ & $43.3 \pm 0.1$ & - & $299.8 \pm 1.0$ & $139.5 \pm 0.3$ & - & $829.1 \pm 5.7$ & $467.4 \pm 2.5$ & - \\
\hline 10 & $126.4 \pm 4.2$ & $46.5 \pm 1.0$ & $72.7 \pm 3.0$ & $342.3 \pm 10.8$ & $157.2 \pm 2.5$ & $85.2 \pm 1.0$ & $881.2 \pm 14.8$ & $492.9 \pm 7.1$ & $96.4 \pm 1.6$ \\
\hline 30 & $139.2 \pm 3.4$ & $42.4 \pm 0.1$ & $62.7 \pm 1.3$ & $388.2 \pm 4.1$ & $144.6 \pm 1.9$ & $73.4 \pm 1.1$ & $984.2 \pm 13.3$ & $496.7 \pm 5.2$ & $90.5 \pm 0.6$ \\
\hline 60 & $149.4 \pm 2.0$ & $39.9 \pm 0.6$ & $56.2 \pm 1.3$ & $418.7 \pm 5.7$ & $137.9 \pm 2.2$ & $66.8 \pm 1.5$ & $1109.0 \pm 26.3$ & $466.4 \pm 5.7$ & $80.1 \pm 2.0$ \\
\hline 120 & $157.9 \pm 2.9$ & $37.7 \pm 0.3$ & $51.1 \pm 1.0$ & $452.5 \pm 9.6$ & $127.6 \pm 0.4$ & $58.9 \pm 1.2$ & $1195.2 \pm 20.3$ & $427.9 \pm 7.1$ & $71.2 \pm 0.6$ \\
\hline 300 & $160.0 \pm 0.8$ & $37.2 \pm 0.6$ & $49.9 \pm 0.5$ & $488.4 \pm 1.7$ & $120.3 \pm 0.7$ & $52.5 \pm 0.2$ & $1335.1 \pm 14.6$ & $397.5 \pm 5.2$ & $61.5 \pm 1.1$ \\
\hline
\end{tabular}




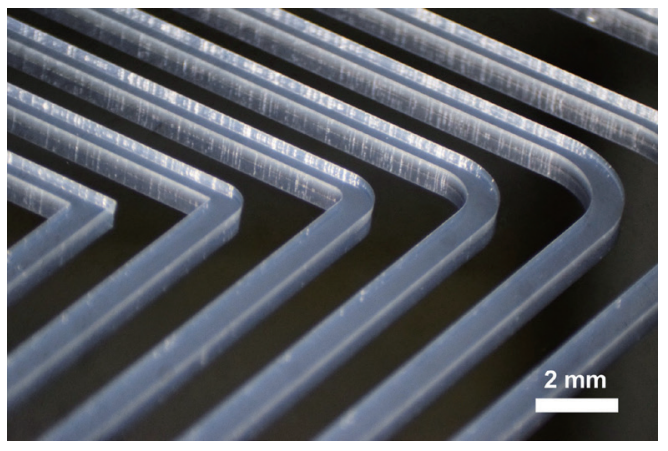

(a)

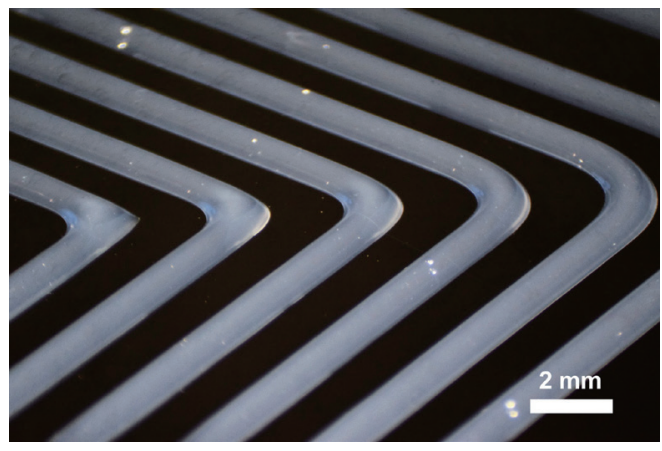

(b)

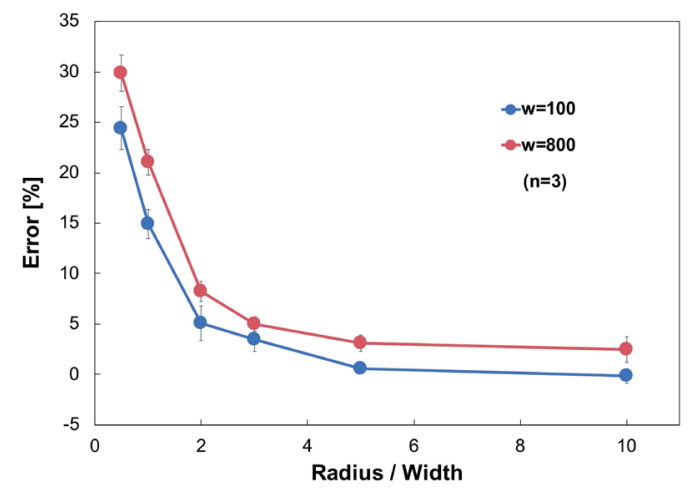

(c)

Fig. 7. (Color online) $(a, b)$ Glue mold before and after reflow. (c) Rate of increase in width of curved section relative to width of straight section. Error bars indicate the standard deviation.

that the cross-sectional shape of the channel changed significantly. For the channel with fillet radius $r=0$ [the leftmost channel in Figs. 7(a) and 7(b)], the errors were $0.65 \%$ and $4.25 \%$ at $w=$ 100 and 800 , respectively, assuming that the ideal width is $\sqrt{2}$ times the width of the straight channel after reflow. The relatively low error in the case of the channel without a fillet was attributed to the fact that the outer line of the corner evolved inward, causing the error to be canceled out. Figure 7(c) shows that the error at the curved portion decreased as the radius of curvature increased. In fact, it was less than $5 \%$ when the radius was more than three times the initial channel width. These results show that no significant deformation of the cross section in the curved portion occurred in channels with sufficient curvature. Therefore, by setting an appropriate fillet radius, this method can be used to fabricate complex flow structures without significant shape changes in the curved area.

\subsection{Demonstration of microvalve and microperistaltic pump}

A microperistaltic pump comprising three microvalves was fabricated as an application of this method to microfluidic devices, and its performance was evaluated. To demonstrate the sealing performance of the channel as a microvalve, a fluorescent dye solution was placed in the flow path of each device, and the fluorescence intensity at the opening and closing of the valve 
was observed. Figure 8(a) shows fluorescence and bright-field images of the open and closed states of the valve. When the pneumatic chambers were pressurized and the diaphragm ascended, the fluorescent dye solution in the valve was excluded, and the fluorescence intensity approached the background value. This indicates that the flow path was completely closed. Figure 8 (b) shows the opening of the valve plotted against the pressure applied to the pneumatic chamber. The average fluorescence intensity in the measured area is indicated as the opening level. All types of valves with different cross sections were closed at a pressure of less than $60 \mathrm{kPa}$. Figure 9 shows the measured flow rates of the pumps. The flow rate was measured using microbeads in the channel as tracers. The flow rate of all devices increased in proportion to the operating frequency up to approximately $20 \mathrm{~Hz}$, and then the flow rate increased gradually. The maximum flow rate was $237 \mu \mathrm{L} / \mathrm{min}$ at $50 \mathrm{~Hz}$, as observed for the Type $\mathrm{C}$ device with the largest channel dimensions. The valves and pumps described in this section are applicable to systems that require high flow rates or the transport of large particles such as cellular tissues.

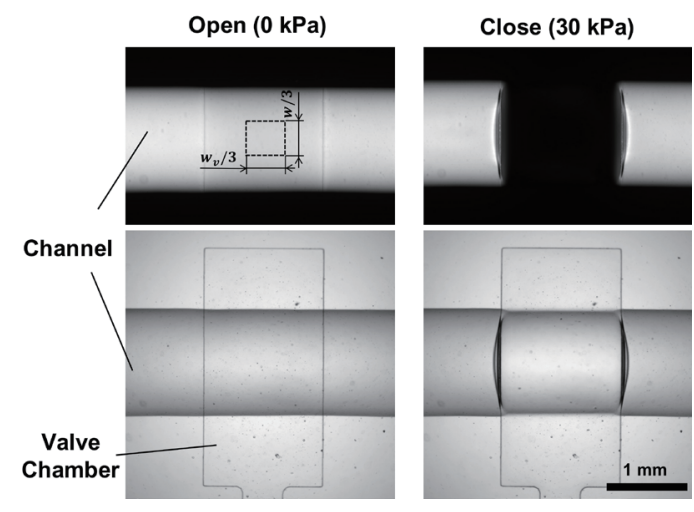

(a)

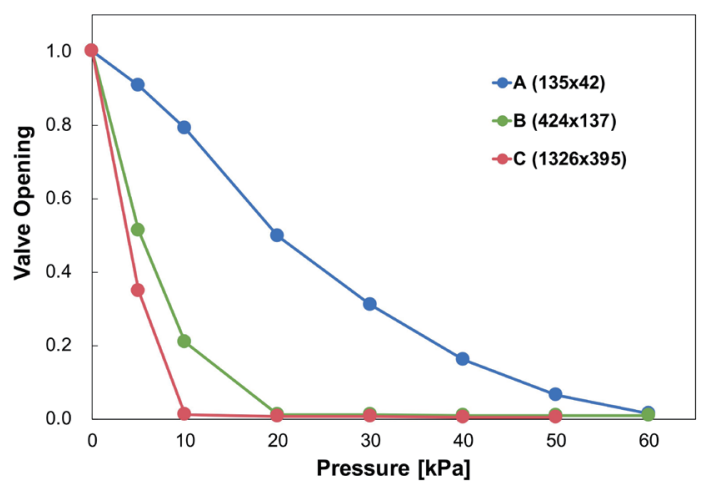

(b)

Fig. 8. (Color online) (a) Photographs of valve opening and closing. Upper photographs show fluorescence images and lower photographs show bright-field images. The measured area of fluorescence intensity, i.e., an area of $w_{v} / 3 \times$ $w / 3$ including the valve center, is shown in the upper-left photograph. (b) Relative opening of valve. The average intensity in the measured area when the applied pressure of $0 \mathrm{kPa}$ was set to $1.0 \mathrm{kPa}$ is shown. Numbers in the legend represent the dimensions of the rounded channel (width $\times$ height) $[\mu \mathrm{m}]$.

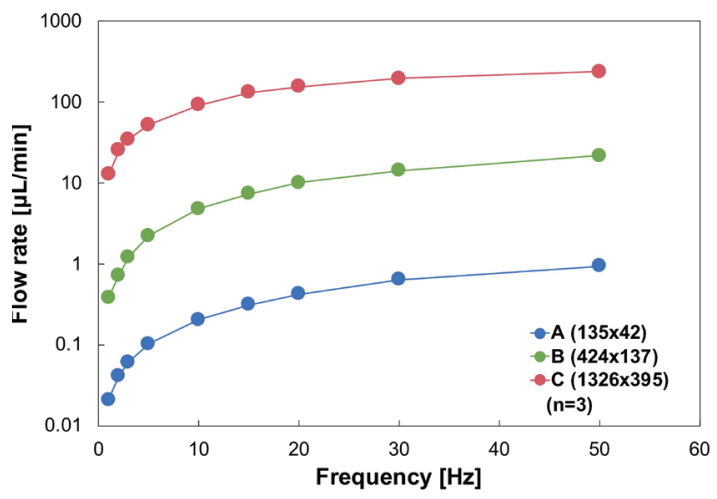

Fig. 9. (Color online) Flow rate of microperistaltic pump vs operating frequency. Numbers in the legend represent the dimensions of the semicircular channel (width $\times$ height) $[\mu \mathrm{m}]$. Error bars indicate the standard deviation. 


\section{Conclusion}

We reported a thermal reflow process using EVA-based hot-melt glue and VAM. This method can be used to fabricate reflowable mold structures with a scale of several hundred micrometers by patterning EVA glue on substrates using PDMS molds fabricated from SU-8 master molds. We first patterned a straight channel on a silicon wafer using VAM and then executed the reflow process. PDMS was cast using fabricated molds, the cross section was observed, and a rounded cross section was formed. The CV of the shape change for each heating time was less than $5 \%$, and it was confirmed that the cross-sectional shape can be controlled via the heating time. In addition, the maximum height of the rounded channel achieved in this experiment was $503 \mu \mathrm{m}$ at a width of $996 \mu \mathrm{m}$. Subsequently, a channel structure that can be bent at right angles was fabricated to evaluate the ability of the process for complex patterning. No significant deformation of the pattern due to the shape change of the glue was observed. A microperistaltic pump comprising three valves was fabricated as an application of this method. The fluorescence observation of the valves and the measurement of the pumping flow rate indicated that the proposed method can be applied to fabricate microfluidic devices. This method can be used to fabricate relatively large-scale channel structures. Moreover, the mold surface afforded is smoother than that obtained by micromilling. In addition, the PDMS and EVA molds can be used repeatedly. The method can be performed using a hot plate and a small vacuum pump; furthermore, it does not require surface treatment on the PDMS mold. Therefore, it can be used as a low-cost and simple process for fabricating structures on the scale of several hundred micrometers.

\section{Acknowledgments}

This work was supported by JKA and its promotion funds from KEIRIN RACE.

\section{References}

M. K. Raj and S. Chakraborty: J. Appl. Polym. Sci. 137 (2020) 48958.

Y. Xia and G. M. Whitesides: Angew. Chem. Int. Ed. 37 (1998) 550.

J.-Y. Qian, C.-W. Hou, X.-J. Li, and Z.-J. Jin: Micromachines 11 (2020) 172.

A. T. H. Lau, H. M. Yip, K. C. C. Ng, X. Cui, and R. H. W. Lam: Micromachines 5 (2014) 50.

Y. Chen, Y. Tian, Z. Xu, X. Wang, S. Yu, and L. Dong: Appl. Phys. Lett. 109 (2016) 143510.

H.-P. Chou, M. A. Unger, and S. R. Quake: Biomed. Microdevices 3 (2001) 323.

M. A. Unger, H. P. Chou, T. Thorsen, A. Scherer, and S. R. Quake: Science 288 (2000) 113.

P. M. Fordyce, C. A. Diaz-Botia, J. L. DeRisi, and R. Gomez-Sjoberg: Lab Chip 12 (2012) 4287.

S. Okamoto and Y. Ukita: Micro Nano Eng. 8 (2020) 100055.

10 Y. Li, C. Pan, Y. Li, E. Kumacheva, and A. Ramachandran: Biomed. Microdevices 19 (2017) 82.

11 Y.-C. Chen, G.-Y. Chen, Y.-C. Lin, and G.-J. Wang: Microfluid. Nanofluid. 9 (2010) 585.

12 D. Kim, W. S. Lee, S. Shin, H. S. Rho, J. Dai, J. Y. Yun, and J. W. Hong: Sci. Adv. Mater. 6 (2018) 2428.

13 L. M. Fidalgo and S. J. Maerkl: Lab Chip 11 (2011) 1612.

14 T. Thorsen, S. J. Maerkl, and S. R. Quake: Science 298 (2002) 580.

15 T. S. Santisteban, O. Rabajania, I. Kalinina, S. Robinson, and M. Meier: Lab Chip 18 (2018) 153.

16 M. Kaminaga, T. Ishida, and T. Omata: Micromachines 7 (2016) 224.

17 A. Rammohan, P. K. Dwivedi, R. Martinez-Duarte, H. Katepalli, M. J. Madou, and A. Sharma: B Chem. 153 (2011) 125. 
18 E. G. B. M. Bossink, A. R. Vollertsen, L. I. Seggerink, and M. Odijk: 23rd Int. Conf. Miniaturized Systems for Chemistry and Life Sciences ( $\mu$ TAS, 2019) 664.

19 J. Hansson, M. Hillmering, T. Haraldsson, and W. V. D. van der Wijngaart: Lab Chip 16 (2016) 1439.

20 C. K. Oh, S. W. Lee, and O. C. Jeong: Microfluid. Nanofluid. 19 (2015) 1091.

21 N. L. Jeon, I. S. Choi, B. Xu, and G. M. Whitesides: Adv. Mater. 11 (1999) 946.

22 E. Kim, Y. Xia, and G. M. Whitesides: J. Am. Chem. Soc. 118 (1996) 5722.

23 E. Kim, Y. Xia, and G. M. Whitesides: Nature 376 (1995) 581.

24 S.-J. Ahn and J. Moon: J. American Ceramic Society 88 (2005) 1171.

25 H. Yang, P. Deschatelets, S. T. Brittain, and G. M. Whitesides: Adv. Mater. 13 (2001) 54.

26 S. Baig, G. Jiang, Q. Sun, and M. R. Wang: J. Europ Opt. Soc. Rap Public 8 (2013) 13076.

27 A. Flores, S. Song, S. Baig, and M. R. Wang: IEEE Photon. Technol. Lett. 20 (2008) 1246.

28 D. C. Duffy, J. C. McDonald, O. J. A. Schueller, and G. M. Whitesides: Anal. Chem. 70 (1998) 4974. 